\title{
Image-Based Quantitative Analysis of Gold Immunochromatographic Strip via Cellular Neural Network Approach
}

\author{
Nianyin Zeng, Zidong Wang*, Bachar Zineddin, Yurong Li, Min Du, Liang Xiao, Xiaohui Liu, \\ and Terry Young
}

\begin{abstract}
Gold immunochromatographic strip assay provides a rapid, simple, single-copy and on-site way to detect the presence or absence of the target analyte. This paper aims to develop a method for accurately segmenting the test line and control line of the gold immunochromatographic strip (GICS) image for quantitatively determining the trace concentrations in the specimen, which can lead to more functional information than the traditional qualitative or semi-quantitative strip assay. The canny operator as well as the mathematical morphology method is used to detect and extract the GICS reading-window. Then, the test line and control line of the GICS reading-window are segmented by the cellular neural network (CNN) algorithm, where the template parameters of the CNN are designed by the switching particle swarm optimization (SPSO) algorithm for improving the performance of the CNN. It is shown that the SPSO-based CNN offers a robust method for accurately segmenting the test and control lines, and therefore serves as a novel image methodology for the interpretation of GICS. Furthermore, quantitative comparison is carried out among four algorithms in terms of the peak signal-to-noise ratio. It is concluded that the proposed CNN algorithm gives higher accuracy and the CNN is capable of parallelism and analog VLSI (very-large-scale integration) implementation within a remarkably efficient time.
\end{abstract}

Keywords - Gold immunochromatographic strip; cellular neural networks; switching particle swarm optimization; mathematical morphology; image segmentation.

\section{INTRODUCTION}

Cellular Neural Network (CNN), proposed by $[3,4]$ in 1988 , is a large-scale nonlinear analog circuit capable of

Copyright (c) 2010 IEEE. Personal use of this material is permitted. However, permission to use this material for any other purposes must be obtained from the IEEE by sending a request to pubspermissions@ieee.org.

This work was supported in part by the Natural Science Foundation of China under Grants 61104041 and 61329301, International Science and Technology Cooperation Project of Fujian Province of China under Grant 2009I0016, the Jiangsu Provincial Key Laboratory of E-business at Nanjing University of Finance and Economics of China under Grant JSEB201301, the Royal Society of the U.K., and the Alexander von Humboldt Foundation of Germany.

N. Zeng, Y. Li and M. Du are with the College of Electrical Engineering and Automation, Fuzhou University, Fuzhou 350002, P. R. China, and also with the Fujian Key Laboratory of Medical Instrumentation and Pharmaceutical Technology, Fuzhou 350002, P. R. China.

Z. Wang, B. Zineddin, X. Liu and T. Young are with the Department of Information Systems and Computing, Brunel University, Uxbridge, Middlesex, UB8 3PH, United Kingdom. Email address: Zidong. Wang@brunel.ac.uk

L. Xiao is with the School of Computer Science and Technology, Nanjing University of Science and Technology, Nanjing 210094, China.

${ }^{*}$ Corresponding author. processing a large amount of information in parallel and in real time. The general idea of CNN is to combine the architecture of cellular automata with neural networks. In the past decade, CNNs have gained much attention with wide applications in image and signal processing, robotics and pattern recognition. It is remarkable that CNN allows for efficient VLSI (very-large-scale integration) implementation of analogue, array-computing structures [32]. Recently, the CNN algorithm has been applied for the GICS (Gold immunochromatographic strip) image segmentation via adaptively setting the threshold value $I$ in Ref. [36]. It is shown that the CNN offers a robust method for the interpretation of the GICS.

The GICS is a new lateral-flow immunoassay technique that uses a cellulose membrane as the carrier and a colloidal gold-labeled antigen (or antibody) as the tracer. The GICS assay has recently attracted considerable research attention for qualitative and semi-quantitative monitoring in resource-poor or non-laboratory environments owing to its attractive properties such as short analysis time, ease of use, low cost, high sensitivity, good specificity and satisfactory stability [23, 25,31]. Especially, researchers have been focusing on not only the improvement of the biochemical properties of the strips via material selection (see e.g. $[14,17])$ but also the establishment of accurate mathematical models for lateral flow immunoassay in order to optimize strip performance for the purpose of quantification (see e.g. [24,33-35,37]). On the other hand, the quantitative instrument of lateral flow immunoassay has been developed that has stirred a great deal of research attention (see e.g. $[5,8,9,16,18,19,28]$ ).

Up to now, most available methods for developing quantitative instruments involve the reflectance photometers for acquiring immunochromatographic strip signals, see e.g. $[8,9,19]$. In this case, mechanical scanning devices are needed which might cause slow operation with bulky style. An alternative way is to develop image-based instruments which are both cost effective and numerically efficient, see e.g. $[5,16,18,28]$. Nevertheless, it is quite challenging to choose a suitable image process technology. In particular, an efficient image segmentation algorithm is of vital importance to the immunochromatographic strip image analysis. In the literature, the Otsu threshold selection method, the fuzzy c-means (FCM) clustering algorithm and genetic fast FCM algorithm have been employed to segment the control and test lines of GICS $[5,18,28]$. However, the background 
in the reading-windows of the GICS image always contains certain level of noises on the strip that might result from the influence of temperature, the humidity as well as the non-uniform penetration of liquid and colloidal gold.

For images of low concentration with strong noisy background, it is difficult to infer a threshold because of the possible low signal-noise-ratio (i.e. part of the background intensity is much larger than the signal), which is actually the main drawback of the threshold method and the FCM clustering algorithm. Meanwhile, the genetic fast FCM clustering algorithm has been applied in GICS in order to cope with the local optimum problem of FCM algorithm [18]. This algorithm performs well but there is a need to run it in every segmentation, which gives rise to a time-consuming computation issue. The CNN algorithm proposed in [36] can avoid the limitations of the aforementioned segmentation techniques while still keeping satisfactory accuracies. However, by analyzing the dynamic properties of $\mathrm{CNN}$, we are only able to get the range of the template parameters. At this stage, it is still very difficult to obtain the best (optimal) template parameters that would give rise to optimal performance of the CNN [10]. Thus, we hope to find an approach in this paper to optimize the template parameters of the CNN for improving its performance.

There are many global search algorithms available in the literature, for example, genetic algorithm, evolutionary algorithm and Particle Swarm Optimization (PSO) algorithm. For the purpose of optimizing the template parameters of the $\mathrm{CNN}$, the suitable algorithm should have the following characteristics: 1) no requirement on the ranking of solutions; 2) strong influence of best solution on the population; 3) satisfactory continuity of search; and 4) ability to reach good solution without local search. In views of the listed characteristics, the PSO algorithm stands out as an ideal candidate, see [13] for the comparison between different search algorithms. The PSO algorithm developed by Kennedy and Eberhart [12] stimulates the social behaviors of birds blocking or fish schooling, etc. It has been successfully applied in a variety of fields due to its effectiveness in performing difficult optimization tasks and its convenience for implementation with fast convergence to a reasonably good solution $[12,26,29]$. In [29], a switching PSO algorithm has been developed that introduces a mode-dependent velocity updating equation with Markovian switching parameters in order to overcome the contradiction between the local search and global search. The switching PSO algorithm developed in [29] can avoid the local search stagnating in a local area (hence wasting more time on an invalid search), and also lead the swarm move to a more potential area quickly which helps to obtain a global search greatly. Thus, the main intention of this paper is therefore to optimize the template parameters of CNN by the switching particle swarm optimization (SPSO) algorithm for accurately segmenting the test and control lines of GICS image.

It should be pointed out that, the GICS image itself exhibits the following distinguishing features that add to the difficulties in its processing [36]. First, in order to improve the efficiency and effectiveness, we only focus on the GICS reading-windows with the test and control lines appearing on the nitrocellulose membrane. Thus, it would be better to obtain the reading-window through segmentation. Second, the test and control lines may appear blurred, uncertain, and mixed with background because they are usually made/smeared by a roller in a non-uniform way. Furthermore, when the testing liquid (e.g. urine, blood, serum) is added to the strip, there might be a lot of interference signals appearing on the strip. With hope to address the above listed challenges, we aim to develop a SPSO-based cellular neural network in combination with the mathematical morphology method in order to achieve the segmentation of the GICS image. The proposed algorithm offers a novel image methodology for interpreting gold immunochromatographic strip.

The main contribution of this paper is mainly threefold. 1) A novel image segmentation methodology for the interpretation of gold immunochromatographic strip is developed, which is based on the CNN approach for segmenting the reading-window image extracted via canny operator as well as mathematical morphology algorithm. 2) The template parameters of the CNN are designed by incorporating the switching PSO algorithm so that the CNN algorithm can offer a robust way for accurately extracting the test and control lines. 3) Our results show that the proposed algorithm gives high accuracy from the segmentation performance, the feature parameter as well as the quantitative comparison in terms of the peak signal-to-noise ratio. Moreover, the proposed method can be easily adapted for the interpretation of gold immunochromatographic strip.

The rest of this paper is organized as follows. The gold immunochromatographic strip assay is introduced in Section II. In Section III, the canny operator as well as mathematical morphology method for the GICS reading-widow extraction are summarized, and the SPSO-based CNN approach for the segmentation of GICS reading-window images are described. The results of image segmentation by the proposed method are discussed in Section IV and the overall performance is also demonstrated. Finally, concluding remarks are given in Section V.

\section{The Gold Immunochromatographic Strip IMAGE AND PROBLEM Formulation}

Gold immunochromatographic strip is based on colloidal gold labeled and chromatographic technology (Fig. 1). A typical immunochromatographic strip design utilizes the specific interaction between antigens and antibodies. In this paper, we focus on the sandwich format of GICS where one antibody is immobilized on the nitrocellulose membranes or other solid phase. The other antibody is labeled with colloidal gold. The labeled antibody is dried on a piece of low protein binding material in conjugate pad. When the specimen passes through and re-wet the material that is dried with the antibody conjugate, it will release the antibody conjugate. If the sample contains the antigen to be detected, the antigen will react with antibody conju- 
gate and form an antigen-antibody conjugate compound. The compound continues to move by capillary action to the membrane where the capture antibody is embedded. The antibody will capture the antigen-antibody conjugate to form a sandwich type compound. This sandwich type compound will stay on the membrane and form a visible red or purple red color band or spot at the test and the control lines, otherwise the redundant compound goes forward to the absorbent pad. After the antigen-antibody reactions,

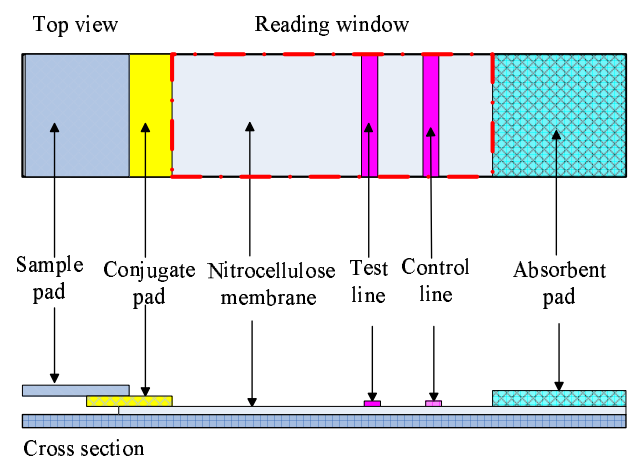

Fig. 1. The schematic structure of the gold immunochromatographic strip

the red or purple red color caused by the accumulation of the colloidal gold at the test and the control lines will appear on the membrane. Monitoring the strength and area of the red color band provides the basis for the quantitative determination of the target molecule. Therefore, the concentration of the measured substance can be determined.

In this study, the gold immunochromatographic strip of human chorionic gonadotropin (hCG) is used as a model. The quantitative tracking result of hCG in serum or urine can provide more useful information in ectopic pregnancy differentiation and in fetal Down syndrome screening test. The GICS images taken from the ten specimens are shown in Fig. 2. The main purpose of this paper is to accurately extract the test line and control line of the GICS images.

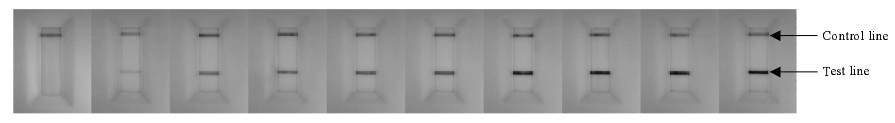

Fig. 2. Images of gold immunochromatographic strip of different concentration(from the left side: $0,10,35,75,100,150,200,300$, 400, 500mIU/ml).

\section{Process of Gold Immunochromatographic STRIP IMAGE}

In this section, for the convenience of the readers, we introduce the canny operator as well as mathematical morphology method for the GICS reading window extraction, and then discuss the cellular neural networks for the filtering and segmentation issues of GICS reading-window images described in (Fig. 3).

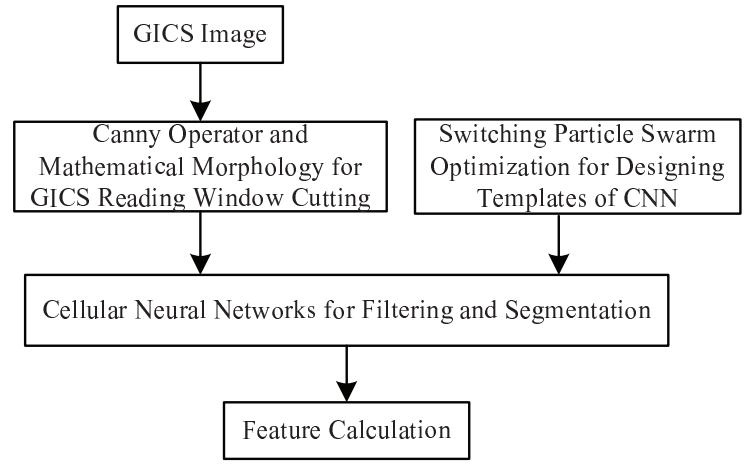

Fig. 3. Image processing for gold immunochromatographic strips

\section{A. Canny Operator and Mathematical Morphology for GICS Reading Window Cutting}

Canny operator [1] is an excellent edge-detection method which has preferable anti-noise ability. However, the edge got by this method is not a consecutive curve. Thus, after obtaining the edge by canny operator, the mathematical morphology is applied to extract the GICS reading window.

Mathematical morphology is nonlinear image processing methodology that is based on the application of lattice theory to spatial structures $[6,27]$. It has become popular in the image processing field due to its rigorous mathematical description and its proven applicability in a number of imaging problems including noise elimination, feature extraction and image compression. The basic operations of mathematical morphology is erosion and dilation, which are defined as

$$
\begin{aligned}
(f \ominus b)(s, t)=\quad & \max \{f(s+x, t+y)+b(x, y) \mid \\
& (s+x, t+y) \in D_{f} \\
& \text { and } \left.(x, y) \in D_{b}\right\} \\
(f \oplus b)(s, t)= & \max \{f(s-x, t-y)+b(x, y) \mid \\
& (s-x, t-y) \in D_{f} \\
& \text { and } \left.(x, y) \in D_{b}\right\}
\end{aligned}
$$

where $D_{f}$ is the domain of the gray-scale image and $D_{b}$ is the domain of the structuring element. $\ominus$ and $\oplus$ are expressed as erosion and dilation operators, respectively. Based on these two operators, the opening and the closing are defined by

$$
\begin{aligned}
& f \circ b=(f \ominus b) \oplus b \\
& f \bullet b=(f \oplus b) \ominus b
\end{aligned}
$$

The opening (or closing) simplifies by removing the bright (or dark) components that do not fit within the structuring element.

\section{B. Cellular Neural Network}

Cellular Neural Network (CNN), proposed in $[3,4]$ in 1988 , is a large-scale nonlinear analog circuit which processes signals in real time. The basic circuit unit of cellular neural networks is called a cell, see Fig. 4. It contains 
linear and nonlinear circuit elements, which are typically linear capacitors, linear resistors, linear and nonlinear controlled sources, and independent sources. The structure of cellular neural networks is similar to that found in cellular automata; namely, any cell in a cellular neural network is connected only to its neighbor cells. The adjacent cells can interact directly with each other. Cells not directly connected together may affect each other indirectly because of the propagation effects of the continuous-time dynamics of cellular neural networks.

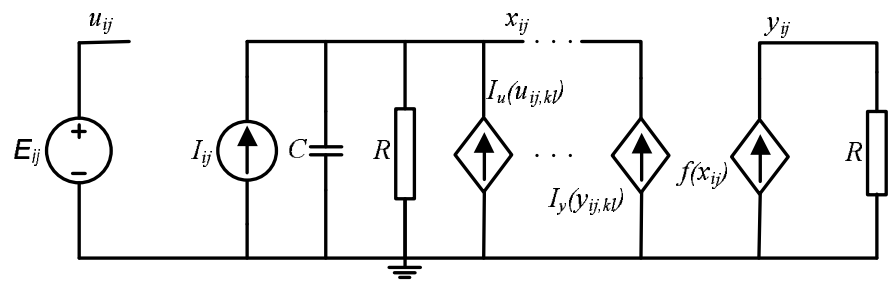

Fig. 4. An example of a cell circuit

Consider a two-dimension $M \times N$ cellular neural network, having $M \times N$ cells arranged in $M$ rows and $N$ columns. We denote the cell located in the $i$ th row and $j$ th column by $C_{i j}$. In addition, the r-neighborhood (the sphere of influence) in CNN is defined as follows:

$$
\begin{aligned}
N_{r(i j)}= & \left\{C_{k l} \mid \max (|k-i|,|l-j|) \leq r,\right. \\
& 1 \leq k \leq M, \quad 1 \leq l \leq N\}
\end{aligned}
$$

where $r$ represents the $r$-neighborhood radius of cell $C_{i j}$ and is a positive integral number.

The circuit equations of a cell dynamics are described by the following nonlinear ordinary differential equations:

State equation:

$$
\begin{aligned}
C \frac{d}{d t} x_{i j}(t) & =-\frac{1}{R} x_{i j}(t)+\sum_{k l \in N_{r}} A_{i, j ; k, l} y_{k l}(t) \\
& +\sum_{k l \in N_{r}} B_{i, j ; k, l} u_{k l}(t)+I_{i j}
\end{aligned}
$$

Output equation:

$$
y_{i j}(t)=\frac{1}{2}\left(\left|x_{i j}(t)+1\right|-\left|x_{i j}(t)-1\right|\right)
$$

Constrained conditions:

$$
\begin{array}{r}
\left|x_{i j}(0)\right| \leq 1,\left|u_{i j}\right| \leq 1 \\
1 \leq i \leq M, 1 \leq j \leq N
\end{array}
$$

where $x_{i j}, u_{i j}$ and $y_{i j}$ are the state, input and output voltages of the specific $\mathrm{CNN}$ cell $C_{i j}$, respectively. The state and output vary in time, the input is static (timeindependent), $i j$ refers to grid point associated with a cell on a $2 D$ grid, and $k l \in N_{r}$ is a grid point in the neighborhood within a radius $r$ of the cell $C_{i j}$. Term $A_{i j, k l}$ represents the linear feedback, $B_{i j, k l}$ is the linear control, while
$I_{i j}$ is the cell current (also referred to as bias or threshold) which could be space and time variant. The time constant of a CNN cell is determined by the linear capacitor $(C)$ and the linear resistor $(R)$ and it can be expressed as $\tau=R C$. A CNN cloning template, the program of the CNN array, is given with the linear and nonlinear terms completed by the cell current. Furthermore, a significant theorem for CNN has been given in [4], i.e., if the circuit parameters satisfy

$$
A_{i j}>\frac{1}{R}
$$

then

$$
\lim _{t \rightarrow \infty}\left|x_{i j}(t)\right|>1, \lim _{t \rightarrow \infty}\left|y_{i j}(t)\right|= \pm 1 .
$$

The above result guarantees that our cellular neural networks have binary-value outputs. This property is crucial for solving classification problems in image processing applications.

Remark 1: In practice, as the digital image is always of the discrete-time nature, the differential equation of $\mathrm{CNN}$ state equation can be rewritten as the difference one when the CNN is applied in the image processing [3]. In general, we let $\tau=1(R=1, C=1)$, so the difference equation of $\mathrm{CNN}$ can be seen as follows:

$$
x_{i j}(t+1)=\sum_{k l \in N_{r}} A_{i, j ; k, l} y_{k l}(t)+\sum_{k l \in N_{r}} B_{i, j ; k, l} u_{k l}(t)+I_{i j}
$$

Thus, we only need to set the feedback template $A$, control template $B$ and the threshold template $I$ when the CNN is used for image processing.

Remark 2: Assuming the r-neighborhood of CNN in this paper is 3 , that is to say, each cell only connected to $3 \times 3$ neighbors. Thus, the template set contains 19 coefficients (feedback template $A$ : $a_{1}, a_{2}, \ldots, a_{9}$, control template $B$ : $b_{1}, b_{2}, \ldots, b_{9}$ and threshold template $\left.I\right)$. However, we can assume the template parameters $a_{1}=a_{3}=a_{7}=a_{9}, a_{2}=$ $a_{4}=a_{6}=a_{8}, b_{1}=b_{3}=b_{7}=b_{9}, b_{2}=b_{4}=b_{6}=b_{8}$ because of the symmetry property of CNN template [4]. The template set can be seen as follows:

$$
\begin{gathered}
A=\left[\begin{array}{lll}
a_{1} & a_{2} & a_{3} \\
a_{4} & a_{5} & a_{6} \\
a_{7} & a_{8} & a_{9}
\end{array}\right]=\left[\begin{array}{lll}
a_{1} & a_{2} & a_{1} \\
a_{2} & a_{5} & a_{2} \\
a_{1} & a_{2} & a_{1}
\end{array}\right] \\
B=\left[\begin{array}{lll}
b_{1} & b_{2} & b_{3} \\
b_{4} & b_{5} & b_{6} \\
b_{7} & b_{8} & b_{9}
\end{array}\right]=\left[\begin{array}{lll}
b_{1} & b_{2} & b_{1} \\
b_{2} & b_{5} & b_{2} \\
b_{1} & b_{2} & b_{1}
\end{array}\right]
\end{gathered}
$$

However, by analyzing the dynamic properties of CNN we can only get the range of the template parameters. Unfortunately, it is still very difficult to choose the best optimal template parameters which results in limiting the performance of the CNN [10]. Thus, it is clear from (13)-(14) that what we need to do is to find a way to optimize the template parameters: $a_{1}, a_{2}, a_{5}, b_{1}, b_{2}, b_{5}, I$.

\section{Switching Particle Swarm Optimization Algorithm for Designing Cloning Templates of CNN}

PSO is a popular stochastic optimization algorithm proposed by Kennedy and Eberhart in 1995 [12]. The main 
idea of PSO algorithm was based on the simulation of simplified social models such as bird flocking and fish schooling. In PSO [29], a swarm consists of $S$ particles moving around in a $D$-dimensional search space. The position of the $i$ th particle is denoted by a vector, $x_{i}(k)=\left(x_{i 1}(k), x_{i 2}(k), \cdots, x_{i D}(k)\right)$, where $x_{i n}(k) \in$ $\left[x_{\min , n}, x_{\max , n}\right](1 \leq n \leq D)$ with $x_{\min , n}$ and $x_{\max , n}$ being lower and upper bounds for the $n$th dimension, respectively. During the search process, the particle successively adjusts its position towards the global optimum according to the two factors: the best position encountered by itself $(p b e s t)$ denoted as $p_{i}=\left(p_{i 1}, p_{i 2}, \cdots, p_{i D}\right)$ and the best position in the whole swarm (gbest) denoted as $p_{g}=\left(p_{g 1}, p_{g 2}, \cdots, p_{g D}\right)$. The velocity of the $i$ th particle at the $k$ th iteration is represented by $v_{i}(k)=$ $\left(v_{i 1}(k), v_{i 2}(k), \cdots, v_{i D}(k)\right)$, and is limited to a maximum velocity $v_{i, \max }=\left(v_{i \max , 1}, v_{i \max , 2}, \cdots, v_{i \max , D}\right) . r_{1, j}$ and $r_{2, j}$ are two uniform random number samples from $U(0,1)$. The parameters $c_{1}$ and $c_{2}$ are called acceleration coefficients, namely, cognitive and social parameters, respectively. The velocity and position of the particle at next iteration are updated according to the following equations:

$$
\begin{array}{ll}
v_{i}(k+1)=\quad & w v_{i}(k)+c_{1} r_{1}\left(p_{i}(k)-x_{i}(k)\right) \\
& +c_{2} r_{2}\left(p_{g}(k)-x_{i}(k)\right), \\
x_{i}(k+1)=\quad & x_{i}(k)+v_{i}(k+1),
\end{array}
$$

where $w$ is the inertia weight. It is shown that a larger inertia weight tends to facilitate the global exploration and a smaller inertia weight achieves the local exploration to fine-tune the current search area [26].

In this paper, a modified version of the traditional PSO algorithm, namely, switching PSO algorithm [29], is employed to tackle the constrained optimization problem. The basic difference between the traditional PSO and the switching PSO algorithms is that, the contradiction between the local search and global search in the traditional PSO algorithm is removed in the switching PSO algorithm. Generally speaking, in the early search stage, the particle in the swarm should keep its independence and swarm's diversity, which helps to enlarge the search scope and avoid premature problem happening. In the latter stage of the search process, all the swarms may converge to the best particle for getting more accurate solution. In the switching PSO algorithm, a mode-dependent velocity updating equation with Markovian switching parameters is introduced to overcome the contradiction between the local search and global search [29]. The velocity and position of the particle at next iteration are updated with Markovian jumping parameters according to the following equations:

$$
\begin{array}{ll}
v_{i}(k+1)= & w(\xi(k)) v_{i}(k)+c_{1}(\xi(k)) r_{1}\left(p_{i}(k)-x_{i}(k)\right) \\
& +c_{2}(\xi(k)) r_{2}\left(p_{g}(k)-x_{i}(k)\right), \\
x_{i}(k+1)=\quad & x_{i}(k)+v_{i}(k+1),
\end{array}
$$

where $w(\xi(k)), c_{1}(\xi(k))$ and $c_{2}(\xi(k))$ are the inertia weight and acceleration coefficients. All of them are modedependent on a non-homogeneous Markov chain $\xi(k)$ $(k \geq 0)$, which take values in a finite state space $\mathcal{S}=$ $\{1,2, \cdots, N\}$ with probability transition matrix $\Pi^{(k)}=$ $\left(\pi_{i j}^{(k)}\right)_{N \times N} \cdot \pi_{i j}^{(k)} \geq 0(i, j \in \mathcal{S})$ is the transition rate from $i$ to $j$ and $\sum_{j=1}^{N} \pi_{i j}^{(k)}=1$. Usually, in the initiative stage, the inertia weights $w(\xi(k))$ and $c_{1}(\xi(k))$ are larger than those of in the latter stage for maintaining the swarm diverse, while $c_{2}(\xi(k))$ is comparatively smaller than that in the early stage for getting the global optimum more accurately.

In order to maintain a balance between global search and local search, $\Pi^{(k)}$ should be adjusted by the current search information such as the swarm diversity and the current best solution in the swarm. The diversity of a swarm is used to describe the distribution of the each individual particle in a whole field, which can be calculated by the diversitymeasure [29] as follows:

$$
\operatorname{Div}(k)=\frac{1}{S \cdot|L|} \sum_{i=1}^{S} \sqrt{\sum_{d=1}^{D}\left(x_{i d}(k)-\bar{x}_{d}(k)\right)^{2}}
$$

where $S$ is the swarm size, $|L|$ is the length of the longest diagonal in the search space, $D$ is the dimension of the objective problem, $x_{i d}$ is the $d$ th value of the $i$ th particle, while $\bar{x}_{d}$ is the $d$ th value of the average point $\bar{x}$ in the whole swarm that can be computed by:

$$
\bar{x}_{d}(k)=\frac{1}{S} \sum_{i=1}^{S} x_{i d}(k) .
$$

In order to design the template parameters of the CNN for image segmentation by the SPSO algorithm in this paper, the Mean Square Error (MSE) is chosen as the fitness function of the SPSO algorithm. The MSE represents the cumulative squared error between the compressed and the original image. The lower the value of MSE, the lower the error. To compute the MSE, the proposed algorithm first produces a binary mask that classifies the image pixels as belonging to either signal (the test and control lines, which is assigned 1) or background (which is assigned 0), then the mean-squared error using the following equation is calculated:

$$
M S E=\frac{\sum_{M, N}\left[I_{1}(m, n)-I_{2}(m, n)\right]^{2}}{M \times N}
$$

where $M$ and $N$ are the numbers of rows and columns in the input images, respectively.

Let $h$ be a coefficient to be used to differentiate the early search stage and late search stage. Here, $h$ is chosen as $h \in\left[\frac{2}{3}, \frac{4}{5}\right] . T_{\max }$ is the largest iterations for the algorithm. For the sake of simplicity, we set $N=2$. The pseudo code of SPSO algorithm for designing the template parameters of CNN is described as follows by above discussion [29]:

Initialize the velocity $v_{i}$ and position $x_{i}(k=0)$.

Set the $w(\xi(0)), c_{1}(\xi(0)), c_{2}(\xi(0)), \Pi^{(0)}$ and initial mode $s=1$. 
while (not satisfying the termination condition)

do

for $i=1$ to particle numbers $S$;

\{

Evaluate fitness of every particle by Eq.(19);

Update the swarm best solution $p_{g}$ and the particle best solution $p_{i}$;

if $\left(0<k<h * T_{\max }\right)$ and diversity $\operatorname{Div}(k)<$ a set value

$[0.3,0.7])$

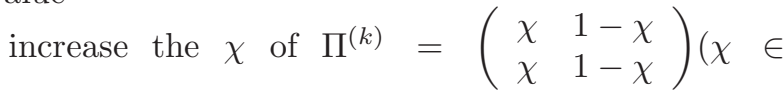

end;

if $\left(k>h * T_{\max }\right)$ and $p_{g}<$ a set value

$$
\Pi^{(k)}=\left(\begin{array}{cc}
\rho & 1-\rho \\
\rho & 1-\rho
\end{array}\right)(\rho \in[0.05,0.2]) ;
$$

end;

Calculate particle new velocity $v_{i}(k+1)$ by Eq.(16);

Calculate particle new position $x_{i}(k+1)$ by Eq.(16);

$\mathrm{k}=\mathrm{k}+1$;

\}

end do;

end;

\section{Simulation Results and Discussion}

\section{A. Performance of SPSO-Based CNN for Image Segmen- tation}

Considering the image characteristics of the gold immunochromatographic strips, especially when the grayscales of the test and control lines are different, we divide the extracted reading-window into two parts. One part includes the control line and its background, and another includes the test line and the corresponding background. According to the above described SPSO algorithm, we set the finite sate space $\mathcal{S}=\{1,2\}$. Here, the mode 1 is set for global search and the mode 2 is for local search. Based on this strategy, let $w_{1}=0.8, c_{11}=2.2$, $c_{21}=1$ in mode 1 and $w_{2}=0.5, c_{12}=1, c_{22}=2$ in mode 2 . The initiation of inputs $\left[a_{1}, a_{2}, a_{5}, b_{1}, b_{2}, b_{5}, I\right]$ are set as $[0.1,1,2,0.1,0.1,0.2,0.5]$. After a few iterations, the SPSO found the feedback template $A$, control template $B$ and the threshold template $I$ of the $\mathrm{CNN}$ algorithm can be described as follows:

$$
\begin{gathered}
A=\left[\begin{array}{lll}
0.1310 & 2.0516 & 0.1310 \\
2.0516 & 2.8190 & 2.0516 \\
0.1310 & 2.0516 & 0.1310
\end{array}\right] \\
B=\left[\begin{array}{lll}
0.1281 & 0.1408 & 0.1281 \\
0.1408 & 0.4043 & 0.1408 \\
0.1281 & 0.1408 & 0.1281
\end{array}\right] \\
I=0.8916
\end{gathered}
$$

In the following, three examples of the segmentation results are shown in Fig. 5, which represent low, middle and high concentration of the specimens respectively.

From Fig. 5, we can see the proposed method can accurately extract the test and control lines of the GICS images

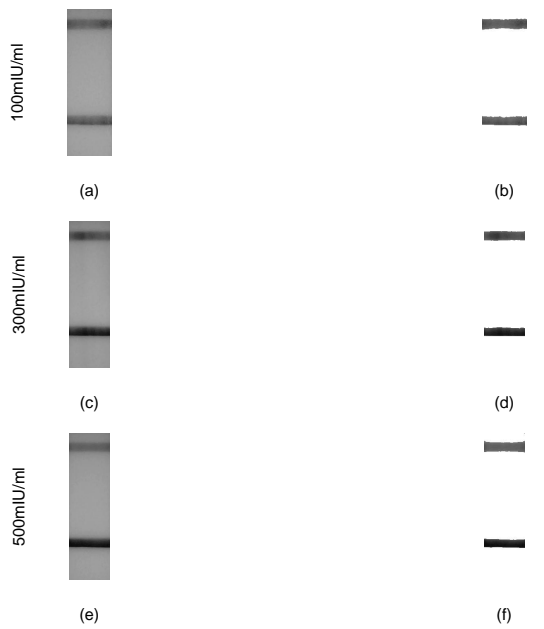

Fig. 5. Three examples of the segmentation results for different concentration of the specimens. Left column: The result of readingwindow extraction by canny operator and mathematical morphology; Right column: The result of the SPSO-based CNN for image segmentation.

which obtained by the low, middle and high concentration of $\mathrm{hCG}$ in the specimens.

\section{B. Feature Calculation and Line Fitting}

In order to get the quantitative results of the GICS system, a feature parameter should be selected to evaluate the concentration of the specimens. A relative integral optical density $(R I O D)$ [18] based on the Lambert-Beer Law is introduced to acquire the concentration of the measured substance. The RIOD can be described as follows:

$$
R I O D=\frac{I O D_{T}}{I O D_{C}}=\frac{\sum_{i=1}^{N} \lg \frac{G_{0}}{G_{T^{2}}}}{\sum_{j=1}^{M} \lg \frac{G_{0}}{G_{C^{j}}}}
$$

where $I O D_{T}$ and $I O D_{C}$ represent the reflective integral optical density of the test line and control line, respectively. $G_{T}$ and $G_{C}$ are the gray level of pixel in the test line and control line. $G_{0}$ is the average gray level of the background in the reading-window.

It is obvious that RIOD can cancel out all the influence, that is, the existence of transmission and scattering will decrease the reflective light intensity, and the non-uniform penetration of water, blood, and colloidal gold will cause the background to only partially reflect the incident light. The results of $R I O D$ for different concentration specimens are calculated by Eq.(20), and also are plotted against sample concentrations by a straight line fitted via a least square method to all of the data points shown in Fig. 6 . From the Fig. 6, we can observe good corresponding relationships between the RIOD and the concentration of $\mathrm{hCG}$ via the two CNN algorithms for GICS image segmentation. The correlation coefficient of the SPSO-based CNN method is 0.97182 . Thus, the proposed method could be a novel image methodology for interpretation of GICS. 


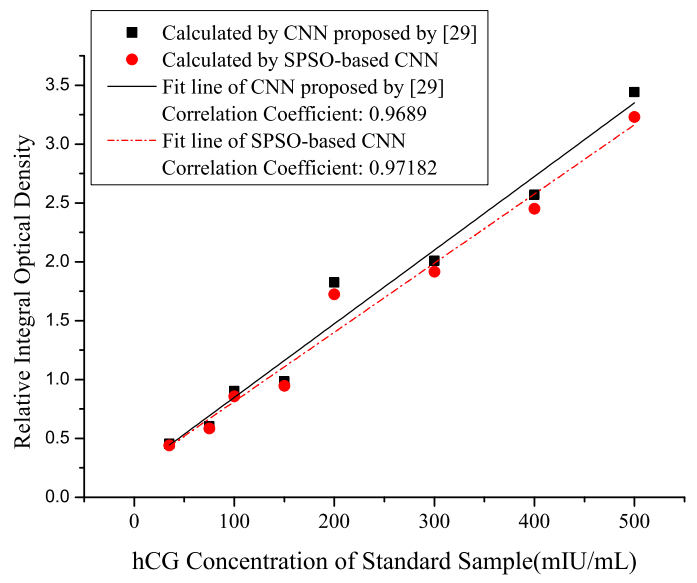

Fig. 6. Correlation between the RIOD and hCG sample concentration, straight line fitted via a least square method to all of the data points.

\section{Evaluation}

In order to quantify the performance of different segmentation methods, a quality measure is required in order to evaluate the validity of the test and control lines. For this purpose, an image quality measurement, known as the peak signal-to-noise ratio (PSNR) [32], is used and the rational is justified as follows.

The Mean Square Error (MSE) and the Peak Signal to Noise Ratio (PSNR) are the two error metrics frequently used to compare image compression quality. The PSNR represents a measure of the peak error. The PSNR is most commonly used as a measure of quality of reconstruction of lossy compression codes (e.g. for image compression). The signal in this case is the original data, and the noise is the error introduced by compression. To compute the PSNR, the proposed algorithm first produces a binary mask that classifies the image pixels as belonging to either signal (the test and control lines, which is assigned 1) or background (which is assigned 0), then the mean-squared error is calculated by Eq.(19), therefore we can obtain the PSNR using the following equation:

$$
P S N R=10 \log \left[\frac{R^{2}}{M S E}\right]
$$

where $R$ is the maximum fluctuation in the input image data type. For example, if the input image has a doubleprecision floating-point data type, then $R$ is 1 . If it has an 8-bit unsigned integer data type, $\mathrm{R}$ is 255 , etc.

The PSNR returns the ratio between the maximum value in the signal and the magnitude of the signal's background noise. The higher the PSNR value the more strongly the binary spot mask fits with the raw image surface. The PSNR is a much better measure of the suitability of a spot mask to the raw image area containing the spot as it is more resilient against large intensity ranges within the spot area compared to the MSE [32].

Fig. 7 shows the PSNR comparison of segmenting images of 8 concentrations of specimens, where the four columns represent the peak signal to noise ratio (PSNR) for the fuzzy c-means (FCM) algorithm, the genetic fast FCM clustering algorithm, the CNN algorithm based on adaptively setting the threshold value and SPSO-based CNN algorithm, respectively. An increase of $20 \mathrm{~dB}$ corresponds to a ten-fold decrease in the MSE difference between two images. Clearly, both of CNN algorithms give a much better segmentation outcome than the FCM method. Furthermore, it is also shown that the proposed SPSO-based CNN algorithm gives higher accuracy than CNN algorithm via adaptively setting the threshold value proposed in [36]. Comparing to the genetic fast FCM clustering algorithm, SPSO-based CNN algorithm has almost the same accuracy. Note that, for the genetic fast FCM clustering algorithm, there is a need to run the genetic algorithm in every segmentation, and this brings a time-consuming computation issue. On the contrary, there is no need to re-apply the SPSO algorithm once the template parameters of CNN algorithm for GICS image segmentation have been designed by the proposed method. As such, the SPSO-based CNN algorithm is more efficient than the genetic fast FCM clustering algorithm. In addition, the $\mathrm{CNN}$ is capable of parallelism and analog VLSI (very-large-scale integration) implementation within a remarkably efficient time.

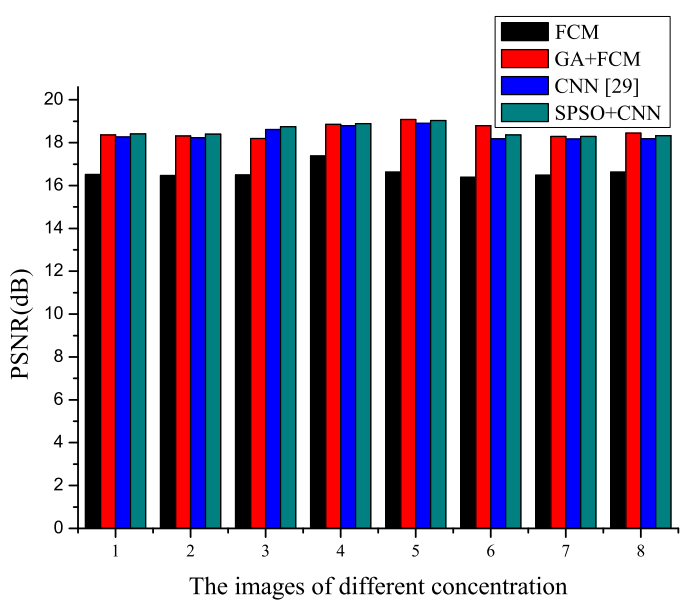

Fig. 7. The PSNR comparison of segmenting images.

\section{Conclusions}

In this paper, we have presented a novel image methodology for interpretation of gold immunochromatographic strip assay. Specifically, the framework consists of two steps, where the first step is to use the canny operator as well as mathematical morphology method for the GICS reading-window extraction and the second step is to apply cellular neural networks for the GICS reading-window filtering and segmentation. The template parameters of CNN are optimized by the switching particle swarm optimization (SPSO) algorithm, where the calculated feature parameter corresponds to the concentration of the target in specimen. Quantitative comparison in terms of the peak signal-to-noise ratio among four algorithms has shown that 
the proposed algorithm gives high accuracy. Furthermore, the cellular neural network is affordable to parallelism and analog VLSI implementation within a remarkably efficient time. In conclusion, the proposed approach adds a novel yet effective way for the quantitative interpretation of immunochromatographic strip. Future research directions would include the modification of the developed algorithms by adopting more advanced image processing techniques $[20,22]$ and data analysis algorithms [2, 7, 11, 15, 21, 38].

\section{REFERENCES}

[1] J. Canny, A computational approach to edge detection, IEEE Transactions on Pattern Analysis and Machine Intelli, vol. 8, pp. 679-698, 1986.

[2] Y. Chen and K. A. Hoo, Stability analysis for closed-loop management of a reservoir based on identification of reduced-order nonlinear model, Systems Science and Control Engineering: An Open Access Journal, vol. 1, no. 1, 2013, pp. 12-19.

[3] L. O. Chua and L. Yang, Cellular neural networks: applications, IEEE Transactions on Circuits and Systems, vol. 35, no. 10, pp. 1273-1290, 1988.

[4] L. O. Chua and L. Yang, Cellular neural networks: theory, IEEE Transactions on Circuits and Systems, vol. 35, no. 10, pp. 1257$1272,1988$.

[5] L. Chuang, J. Hwang, H. Chang, F. Chang and SH. Jong, Rapid and simple quantitative measurement of $\alpha$-fetoprotein by combining immunochromatographic strip test and artificial neural network image analysis system, Clinica Chimica Acta., vol. 348, pp. 87-93, 2004.

[6] Z. Deng, Z. Yin and Y. Xiong, High probability impulse noiseremoving algorithm based on mathematical morphology, IEEE Signal Processing Letters vol. 14, no. 1, pp. 31-34, 2007.

[7] K. El-Tawil and A. Abou Jaoude, Stochastic and nonlinearbased prognostic model, Systems Science and Control Engineering: An Open Access Journal, vol. 1, no. 1, 2013, pp. 66-81.

[8] K. Faulstich, R. Gruler, M. Eberhard and K. Haberstroh, Developing rapid mobile POC systems. Part 1:Devices and applications for lateral-flow immunodiagnostics, IVD Technology, vol. 13, no. 6, pp. 47-53, 2007.

[9] L. Huang, Y. Zhang, C. Xie, J. Qu, H. Huang, and X. Wang, Research of reflectance photometer based on optical absorption, International Journal for Light and Electron Optics, vol. 121, no. 19 , pp. $1725-1728,2010$.

[10] M. Hanggi and G. S. Moschytz, An exact and direct analytical method for the design of optimally robust CNN templates, IEEE Transactions on Circuits System I, Fundamental Theory Application,vol. 46, no. 2, pp. 304-311, 1999.

[11] T. Ishihara and H.-J. Guo, Design of optimal output disturbance cancellation controllers via loop transfer recovery, Systems Science and Control Engineering: An Open Access Journal, vol. 1, no. 1, 2013, pp. 57-65.

[12] J. Kennedy and R. Eberhart, Particle swarm optimization, Proc. IEEE International Conference On Neural Network, pp. 1942-1948, 1995.

[13] V. Kachitvichyanukul, Comparison of three evolutionary algorithms: GA, PSO, and DE, Industrial Engineering 83 Management Systems, vol. 11, no. 3, pp. 215-223, 2013.

[14] J. Kaur, K. Singh, R. Boro, K. Thampi, M. Raje and G. Varshney, Immunochromatographic dipstick assay format using gold nanoparticles labeled protein-hapten conjugate for the detection of atrazine, Environmental Science and Technology, vol. 41, no. 14, pp. 5028-5036, 2007.

[15] Q. Liang, Y.-Z. Wang and Y.-H. Zhang, Resource virtualization model using hybrid-graph representation and converging algorithm for cloud computing, International Journal of Automation and Computing, vol. 10, no. 6, pp. 597-606, 2013.

[16] C. Lin, C. Wu, H. Hsu, K. Li and L. Lin, Rapid bio-test strips reader with image processing technology, Optik, vol. 115, no. 8, pp. 363-369, 2004.

[17] D. Li, S. Wei, H. Yang, Y. Li and A. Deng, A sensitive immunochromatographic assay using colloidal gold-antibody probe for rapid detection of pharmaceutical indomethacin in water samples, Biosensors and Bioelectronics, vol. 24, no. 7, pp. 22772280,2009
[18] Y. Li, N. Zeng and M. Du, A novel image methodology for interpretation of gold immunochromatographic strip, Journal of Computers, vol. 6, no. 3, pp. 540-547, 2011.

[19] J. Li, A. Ouellette, L. Giovangrandi, D. Cooper, A. Ricco and G. Kovacs, Optical scanner for immunoassays with up-converting phosphorescent labels, IEEE Transactions on Biomedical Engineering, vol. 55, no. 5, pp. 1560-1571, 2008.

[20] F. Liu, X. Liu, B. Zhang and J. Bai, Extraction of target fluorescence signal from In vivo background signal using image subtraction algorithm, International Journal of Automation and Computing, vol. 9, no. 3, pp. 232-236, 2012

[21] A. Mehrsai, H. R. Karimi and K.-D. Thoben, Integration of supply networks for customization with modularity in cloud and make-to-upgrade strategy, Systems Science and Control Engineering: An Open Access Journal, vol. 1, no. 1, 2013, pp. 28-42.

[22] P. K. Parlewar, K. M. Bhurchandi. A 4-quadrant curvelet transform for denoising digital images, International Journal of Automation and Computing, vol. 10, no. 3, pp. 217-226, 2013

[23] GA. Posthuma-Trumpie, J. Korf and A. van Amerongen, Lateral flow (immuno)assay: its strengths, weaknesses, opportunities and threats. A literature survey, Analytical and Bioanalytical Chemistry, vol. 393, no. 2, pp. 569-582, 2009.

[24] S. Qian and H. Haim, A mathematical model of lateral flow bioreactions applied to sandwich assays, Analytical Biochemistry, vol. 322, no. 1, pp. 89-98, 2003.

[25] C. Raphael and Y. Harley, Lateral flow immunoassay, Humana Press, 2008

[26] Y. Shi, R C. Eberhart, Empirical study of particle swarm optimization, Proc. 1999 IEEE congress on evolutionary computation, pp. 1945-1950, 1999.

[27] J. Serra, Image Analysis and Mathematical Morphology. London, U.K.: Academic, 1982

[28] E. Sumonphan, S. Auephanwiriyakul and N. Theera-Umpon, Interpretation of nevirapine concentration from immunochromatographic strip test using support vector regression, Proceedings of 2008 IEEE International Conference on Mechatronics and Automation, pp. 633-637, 2008.

[29] Y. Tang, Z. Wang and J. Fang, Parameters identification of unknown delayed genetic regulatory networks by a switching particle swarm optimization algorithm, Expert Systems with Applications, vol. 38, pp. 2523-2535, 2011.

[30] J. Wang, C. Yang and C. Sun, A novel algorithm for edge detection of remote sensing image based on CNN and PSO, 2nd International Congress on Image and Signal Processing, pp. 1-5, 2009.

[31] P. Yager, T. Edwards, E. Fu, K. Helton, K. Nelson, M. R. Tam, B. H. Weigl, Microfuidic diagnostic technologies for global public health, Nature vol. 442 , pp. 412-418, 2006.

[32] B. Zineddin, Z. Wang, Y. Shi, Y. Li, M. Du and X. Liu, A multiview approach to cDNA microarray analysis, International Journal of Computational Biology and Drug Design, vol. 3, no. 2, pp. 91-111, 2010

[33] N. Zeng, Z. Wang, Y. Li, M. Du and X. Liu, Inference of nonlinear state-space models for sandwich-type lateral flow immunoassay using extended Kalman filtering, IEEE Transactions on Biomedical Engineering, vol. 58, no. 7, pp. 1959-1966, 2011.

[34] N. Zeng, Z. Wang, Y. Li, M. Du and X. Liu, A hybrid EKF and switching PSO algorithm for joint state and parameter estimation of lateral flow immunoassay models, IEEE/ACM Transactions on Computational Biology and Bioinformatics, vol. 9, no. 2, pp. 321-329, 2012.

[35] N. Zeng, Z. Wang, Y. Li, M. Du and X. Liu, Identification of nonlinear lateral flow immunoassay state-space models via particle filter approach, IEEE Transactions on Nanotechnology, vol. 11, no. 2, pp. 321-327, 2012.

[36] N. Zeng, Z. Wang, Y. Li and M. Du, Cellular neural networks for gold immunochromatographic strip image segmentation, Lecture Notes in Computer Science vol. 7231, pp. 110-120, 2012.

[37] N. Zeng, Z. Wang, Y. Li, M. Du J. Cao and X. Liu, Time series modeling of nano-gold immunochromatographic assay via expectation maximization algorithm, IEEE Transactions on Biomedical Engineering, vol. 60, no. 12, pp. 3418-3424, 2013.

[38] X. -Y. Zhang and C. -L. Zhou, From biological consciousness to machine consciousness: an approach to make smarter machines, International Journal of Automation and Computing, vol. 10, no. 6, pp. 498-505, 2013. 